Research Paper

\title{
The Impact of Microsatellite Instability Status and Sidedness of the Primary Tumor on the Effect of Cetuximab-Containing Chemotherapy in Patients with Metastatic Colorectal Cancer
}

\author{
Seung Tae Kim, Su-Jin Lee, Jeeyun Lee, Se Hoon Park, Joon Oh Park, Ho Yeong Lim, Won Ki Kang, Young \\ Suk Park ${ }^{凶}$ \\ Division of Hematology-Oncology, Department of Medicine, Samsung Medical Center, Sungkyunkwan University School of Medicine, Seoul, Korea. \\ $\square$ Corresponding author: Young Suk Park, M.D., Ph.D., Division of Hematology/Oncology, Department of Medicine, Samsung Medical Center, Sungkyunkwan University \\ School of Medicine, 81 Irwon-ro, Gangnam-gu, Seoul 06351, Korea, Tel.: +82-2-3410-3451, Fax: +82-2-3410-1754 Email: pys27hmo@skku.edu \\ (c) Ivyspring International Publisher. This is an open access article distributed under the terms of the Creative Commons Attribution (CC BY-NC) license \\ (https://creativecommons.org/licenses/by-nc/4.0/). See http://ivyspring.com/terms for full terms and conditions.
}

Received: 2016.11.10; Accepted: 2017.03.26; Published: 2017.08.23

\begin{abstract}
Background: Colorectal cancer (CRC) has been reconsidered as a heterogeneous disease. Among advances of genomic analysis in CRC, the sidedness of tumors (left-sided colon vs. right-sided colon) and microsatellite instability (MSI)-high $(\mathrm{H})$ tumors have been highlighted.

Methods: We analyzed 153 CRC patients who were available for evaluation of MSI status and had been treated with cetuximab-containing chemotherapy between April 2008 and January 2013. KRAS mutational status was available in all 153 patients, but BRAF mutational status was only available in 72 patients $(47.1 \%)$. We evaluated the impact of microsatellite instability status and location of the primary colon tumor on the effect of cetuximab-containing chemotherapy in patients with metastatic colorectal cancer.

Results: MSI-H was detected in $3.9 \%$ of analyzed patients. Characteristics of patients, with the exception of BRAF mutational status, were generally similar between those with right and left-sided tumors. Right-sided tumors were significantly associated with a BRAF mutation $(p=0.023)$. In addition, patient characteristics with an MSS tumor were not different from those with an MSI-H tumor. For all 153 patients, the most commonly used regimen that included cetuximab was irinotecan alone, irrespective of treatment line. There was no significant difference in treatment efficacy in either RR or disease control rate (DCR) between the MSI-H and MSS groups. There was also no difference in RR and DCR according to the location of the primary tumor (left side vs. right side). No significant difference in PFS was observed between the MSI-H and MSS groups (4.80 months vs. 5.80 months; $p=0.238)$ or the left-side and right-side groups (6.10 months vs. 4.20 months; $p=0.278)$. In a subgroup-analysis of 140 patients with wild-type KRAS, there was no difference in PFS following cetuximab-containing therapy based on MSI status or the location of the primary tumor.

Conclusions: MSI status and the location of the primary tumor were not novel biomarkers for response to cetuximab-containing therapy in metastatic CRC. Further prospective validation of the prognostic or predictive capacity of MSI status and the sidedness of tumors is warranted.
\end{abstract}

Key words: MSI, Sidedness, Cetuximab.

\section{Introduction}

Colorectal cancer $(\mathrm{CRC})$ is the fourth most common cancer in men and third most common cancer in women worldwide [1]. Although gains remain modest, survival has improved in metastatic CRC during the last two decades, partly as a result of the introduction of newly developed molecularly 
targeted agents such as bevacizumab, which targets vascular endothelial growth factor (VEGF-A), and cetuximab and panitumumab, which target epithelial growth factor receptor (EGFR) [2-7]. Cetuximab, a human-mouse chimerized anti-EGFR immunoglobulin G1 monoclonal antibody, has been used to treat wild-type KRAS metastatic CRC with an oxaliplatinor irinotecan-based doublet regimen [8]. Recently, the FIRE-3 trial showed that among patients with wild-type KRAS treated in the cetuximab arm, those with left-sided colon cancer (LC) had significantly improved overall survival (OS), progression-free survival (PFS) and response rate (RR) compared to those with right-sided colon cancer $(\mathrm{RC})$.

CRC develops either sporadically (85\%), as part of a hereditary cancer syndrome (less than 10\%), or against a background of inflammatory bowel disease. It is believed that the adenoma-carcinoma sequence underlies the development of colorectal cancer in most patients, and two distinct pathways have been identified based on microsatellite instability (MSI) and chromosomal instability (CIN) [9-12]. The MSI pathway, which involves failure of the nucleotide mismatch recognition and repair system, is one form of genomic instability [13, 14]. Deficient mismatch repair occurs in approximately $10-15 \%$ of all sporadic CRC [15]. Instability in microsatellite sequences in sporadic colorectal cancer exhibiting MSI, often due to the loss of expression of a mismatch repair gene (most commonly MLH1 and MSH2), is caused by epigenetic silencing [16-18]. MSI-H colorectal cancers are known to bear many features that are generally associated with poor prognosis, including deep tumor invasion and poor histologic differentiation. However, patients with MSI-H tumors have longer overall and cancer-specific survival than stage-matched patients with tumors exhibiting CIN, implying that the pronounced genetic instability of tumor cells with MSI may increase susceptibility to apoptosis $[19,20]$. MSI-H colorectal cancers are more commonly located on the right side [14, 21, 22]. Right and left-sided colon cancer is now recognized to have distinct clinical and genomic features. In addition to MSI status, there are many differences in molecular features between LC and RC.

Herein, we evaluated the impact of MSI status and the sidedness of the primary tumor on the effect of cetuximab-containing chemotherapy in patients with metastatic colorectal cancer.

\section{Materials and Methods}

\section{Patients}

We analyzed 153 CRC patients who were available for evaluation of MSI status and had been treated with cetuximab-containing chemotherapy at Samsung Medical Center, Seoul, Korea between April 2008 and January 2013. The following clinicopathologic characteristics were collected for all 153 patients: age, gender, primary site, number of metastatic sites, Kirsten-ras (KRAS) mutational status, BRAF mutation status and information on chemotherapy. All patients had pathologically or cytologically proven metastatic or recurrent CRC. The definition of LC was cancer in the descending colon, sigmoid colon, and rectum and RC was defined as cancer in the cecum and ascending colon.

\section{Analysis of MSI}

We used the presence of MSI to assess the loss of function of mismatch repair gene activity. Immunohistochemistry (IHC) and/or Multiplex polymerase chain reaction method was used to evaluate the status of MSI. We conducted IHC analysis of MSH2, MSH6, PMS2, and MLH1 proteins with formalin-fixed paraffin-embedded tumor samples. After the tumor area adjacent to normal mucosa and/or lymphocytic infiltration was marked, $4 \mathrm{~mm}$ of paraffinized tissue was removed, and multiple tissue blocks were prepared. Finally, $4-\mu \mathrm{m}$ thick sections were obtained for IHC following standard protocols. The mouse monoclonal antibodies used were anti-MSH2, anti-MSH6, anti-MLH1, and anti-PMS2 (BD Pharmingen). Tumors showing a proportion of stained nuclei higher than $10 \%$ were classified as staining positive; all others were regarded as negative (Figure 1 ).

MSI test was performed by multiplex polymerase chain reaction and analysis with a $3130 \times 1$ genetic analyzer. MSI testing of DNA samples was based on five dinucleotide markers (NR27, NR21, BAT26, BAT25, and NR24). Tumors that showed instability in $\geq 2 / 5$ of markers tested were classified as a high MSI and $1 / 5$ of markers were classified as low MSI. Tumors that showed instability in $0 / 5$ of markers were designated as microsatellite stable (MSS) cancers. Only high MSI cases were considered MSI positive.

\section{Chemotherapy regimens combined with cetuximab}

The chemotherapy regimen to be used with cetuximab was determined by the physician. Typical combinations with cetuximab included oxaliplatin plus intravenous or oral 5-fluorouracil (5-FU) (fluorouracil, leucovorin, and oxaliplatin; [FOLFOX] or capecitabine plus oxaliplatin [XELOX]), and irinotecan plus intravenous or oral 5-fluorouracil (5-FU) (5-fluorouracil, leucovorin, and irinotecan [FOLFIRI]) or capecitabine plus irinotecan [XELIRI]). 
As a single partner of bevacizumab, irinotecan alone was preferred. Chemotherapy was generally repeated every two or three weeks, according to protocol. All tumor measurements were assessed after every three or four cycles of chemotherapy, using computed tomography scan and other tests that were used initially in staging of the tumor.

\section{Statistical analyses}

Descriptive statistics were reported as proportions and medians. Treatment outcomes were response rate (RR) and progression-free survival (PFS). Tumor response was determined according to RECIST ver. 1.0. PFS was defined as the time from the start date of the cetuximab-based chemotherapy to the date of disease progression or death from any cause. PFS was estimated using the Kaplan-Meier method with log-rank analysis. A two-sided p-value of less than 0.05 was considered significant. All analyses were performed using SPSS version 19.0 (SPSS Inc., Chicago, IL, USA). The $\mathrm{X}^{2}$-test or Fisher's exact test was used as appropriate to compare categorical variables. Two-sided null hypotheses of no difference were rejected if p-values were less than 0.05 , or, equivalently, if the $95 \%$ confidence intervals (CIs) of risk point estimates excluded 1. Cox proportional hazards regression modeling was employed in univariate analyses to identify the significant

\section{(A) MSI}

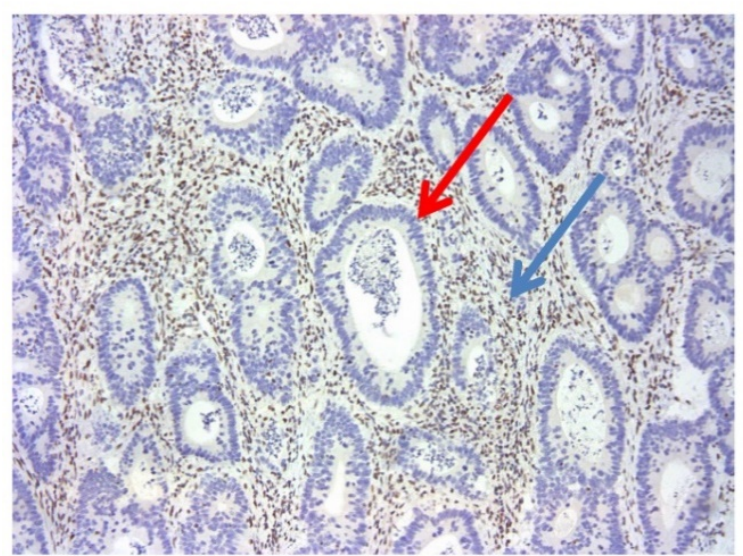

independent prognostic factors of various clinical parameters for survival.

\section{Results}

\section{Patient characteristics}

Among patients with metastatic CRC who were managed at Samsung Medical Center between June 2013 and March 2016, 153 CRC patients with cetuximab-containing therapy and the information for microsatellite instability were analyzed in this study. None of the patients had received cetuximab-containing therapies during their treatment course. MSI-H was detected in 3.9\% of analyzed patients. A summary of patient characteristics according to primary tumor site and MS1 status is shown in Table 1. KRAS mutational status was available in all 153 patients, but BRAF mutational status was only available in 72 patients (47.1\%). Characteristics of patients, except the BRAF mutational status, were generally similar between RC and LC. RCs were significantly associated with a BRAF mutation $(\mathrm{p}=0.023)$.

In addition, characteristics of patients with an MSS tumor were not different from those with an MSI-H tumor. Among the six MSI-H patients, MSI-H tumors were significantly associated with left-sided colon cancer and cancer with wild-type KRAS. Of the three MSI-H patients with available BRAF mutation status, one had a BRAF mutation.

\section{(B) MSS}

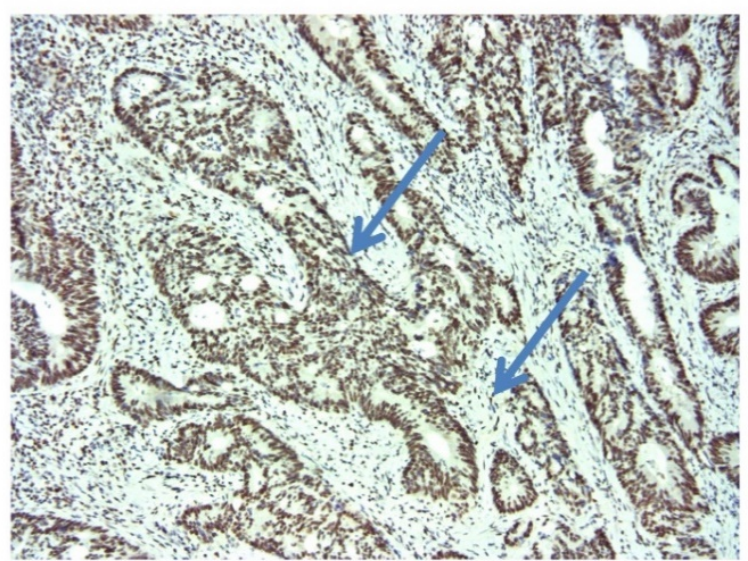

Figure 1. Immunohistochemistry for MLHI (MSI vs. MSS) (x100). Red arrow (negative), and Blue arrow (positive).

Table 1. Patient characteristics

\begin{tabular}{|c|c|c|c|c|c|c|}
\hline \multirow{2}{*}{ Gender } & \multirow{2}{*}{ Rt. Side tumors $(n=29)$} & \multicolumn{2}{|l|}{ Lt. side tumors $(\mathrm{n}=124)$} & \multirow{2}{*}{ MSS (\%) $(\mathrm{n}=147)$} & \multirow{2}{*}{$\operatorname{MSI}(\%)(n=6)$} & \multirow{2}{*}{ p-value } \\
\hline & & & & & & \\
\hline Male & $16(55.2 \%)$ & $75(60.5 \%)$ & 0.676 & $89(60.5)$ & $2(33.3)$ & 0.223 \\
\hline Female & $13(44.8 \%)$ & $49(39.5 \%)$ & & $58(39.5)$ & $4(66.7)$ & \\
\hline
\end{tabular}




\begin{tabular}{|c|c|c|c|c|c|c|}
\hline$\leq 65$ & $19(65.5 \%)$ & $93(75.0 \%)$ & 0.352 & $108(73.5)$ & $4(66.7)$ & 0.659 \\
\hline $65<$ & $10(34.5 \%)$ & $31(25.0 \%)$ & & $39(26.5)$ & $2(33.3)$ & \\
\hline \multicolumn{7}{|c|}{ ECOG performance status } \\
\hline 1 & $29(100.0 \%)$ & $124(100.0 \%)$ & & $147(100.0)$ & $6(100.0)$ & \\
\hline Primary site & - & - & - & & & \\
\hline Left sided colon & - & - & & $121(82.3 \%)$ & $3(50.0 \%)$ & 0.082 \\
\hline Right sided colon & - & - & & $26(17.7 \%)$ & $3(50.0 \%)$ & \\
\hline \multicolumn{7}{|c|}{ No. of metastatic sites } \\
\hline 1 & $11(37.9 \%)$ & $61(49.2 \%)$ & 0.307 & $70(47.6)$ & $2(33.3)$ & 0.685 \\
\hline $1<$ & $18(62.1 \%)$ & $63(50.8 \%)$ & & $77(52.4)$ & $4(66.7)$ & \\
\hline \multicolumn{7}{|c|}{ Treatment line including cetuximab } \\
\hline$\leq 2$ & $11(37.9 \%)$ & $40(32.3 \%)$ & 0.662 & $48(32.7)$ & $3(50.0)$ & 0.401 \\
\hline $2<$ & $18(62.1 \%)$ & $94(67.7 \%)$ & & $99(67.3)$ & $3(50.0)$ & \\
\hline \multicolumn{7}{|l|}{ KRAS status } \\
\hline Wild & $25(86.2 \%)$ & $115(92.7 \%)$ & 0.271 & $134(91.2)$ & $6(100.0)$ & 0.664 \\
\hline Mutant & $4(13.8 \%)$ & $9(7.3 \%)$ & & $13(8.8)$ & $0(0.0)$ & \\
\hline \multicolumn{7}{|l|}{ BRAF status } \\
\hline Wild & 10 & 55 & 0.023 & $63(42.9)$ & $2(33.3)$ & 0.345 \\
\hline Mutant & 4 & 3 & & $6(4.1)$ & $1(16.7)$ & \\
\hline N.E. & 15 & 66 & & $78(53.1)$ & $3(50.0)$ & \\
\hline \multicolumn{4}{|c|}{ Microsatellite instability (MSI) status } & - & - & \\
\hline MSI-H & $3(10.3 \%)$ & $3(2.4 \%)$ & 0.079 & - & - & - \\
\hline MSS & $26(89.7 \%)$ & $121(97.6 \%)$ & & - & - & \\
\hline
\end{tabular}

\section{Treatment efficacy}

For all 153 patients, the most commonly used regimen with cetuximab was irinotecan alone, irrespective of treatment line (Table 2). Cetuximab alone without cytotoxic chemotherapy was the second most common. Among patients overall, none achieved complete response (CR) and 58 achieved partial response, for a response rate (RR) of $37.9 \%$. Table 3 compares the treatment efficacy of cetuximab-containing regimens in all 153 patients according to MSI status (MSI-H vs. MSS). There was no significant difference in treatment efficacy, including RR and disease control rate (DCR), between the two groups. However, there was no difference in RR but significant difference in DCR according to the sidedness of the primary tumor (LC vs. RC). Median PFS for cetuximab-containing chemotherapy was 5.80 (95\% confidence interval [CI], 4.80 to 6.79) (Figure 2A). No significant difference in PFS was observed between the MSI-H and MSS groups (4.80 months; 95\% CI, 0.84 to 8.76 months vs. 5.80 months; $95 \% \mathrm{CI}$, 4.85 to 6.75 months; $\mathrm{p}=0.238$ ) (Figure $2 \mathrm{~B}$ ) or LC and RC groups (6.10 months; $95 \% \mathrm{CI}, 5.32$ to 6.88 months vs. 4.20 months; $95 \% \mathrm{CI}, 2.44$ to 5.96 months; $\mathrm{p}=0.278$ ) (Figure 2C). In a subgroup-analysis of 140 patients with wild-type KRAS, there was no difference in PFS following cetuximab-containing therapy according to MSI status (MSI-H versus vs. MSS) or sidedness of the primary tumor (RC versus, LC) (Figures 3A and 3B).

\section{Prognostic analysis}

Results from the prognostic analysis for cetuximab-containing chemotherapy are shown in Table 4. A univariate analysis found no evidence of an effect of MSI status on PFS after cetuximab-containing chemotherapy (hazard ratio, 1.111; 95\% CI, 0.487 to 2.534; $\mathrm{p}=0.801$ ). Similarly, there was no evidence indicating that sidedness of the primary tumor ( $\mathrm{RC}$ vs. LC) was a prognostic factor for PFS (hazard ratio, 1.322; $95 \% \mathrm{CI}, 0.870$ to $2.008 ; \mathrm{p}=0.191$ ).

Table 2. Chemotherapy regimens including cetuximab.

\begin{tabular}{lll}
\hline Regimen & MSS $(\mathrm{n}=147)$ & MSI $(\mathrm{n}=6)$ \\
\hline FOLFIRI/cetuximab & $16(10.9)$ & $2(33.3)$ \\
FOLFOX/cetuximab & $13(8.8)$ & \\
XELOX/cetuximab & $7(4.8)$ & \\
XELRI/cetuximab & $7(4.8)$ & $3(50.0)$ \\
Irinotecan/cetuximab & $83(56.5)$ & $1(16.7)$ \\
Cetuximab & $21(14.3)$ & \\
\hline
\end{tabular}

\section{Discussion}

The use of next generation sequencing (NGS) for high throughput genomic analysis has accelerated our understanding of the molecular characteristics of CRC [23-26]. Several groups have reported on the molecular classification of CRC using genomic data from NGS; in some cases, these subtypes have provided predictive or prognostic information. Recently, among advances in genomic analysis in CRC, the sidedness of tumors (LC vs. RC) and MSI-H tumors have been highlighted. The present study evaluated the impact of MSI status and sidedness of primary colon cancer on the effect of cetuximab-containing chemotherapy in 153 patients (140 KRAS wild type and 13 KRAS mutation) with metastatic colorectal cancer. PFS following cetuximab-containing therapy was not different 
according to the location of the primary tumor or MSI status. This finding was only consistent in the 140 patients with wild-type KRAS. Thus, MSI status and the sidedness of primary colon cancer might not be novel biomarkers to assign cetuximab-containing therapy in metastatic CRC.

Table 3. Treatment efficacy of regimens containing cetuximab according to microsatellite instability status and location of the primary tumor.

\begin{tabular}{|c|c|c|c|c|c|c|c|}
\hline Response & Overall & MSS (n=147) & MSI $(n=6)$ & p-value & Rt side $(\mathrm{N}=29)$ & Lt side $(\mathrm{N}=124)$ & p-value \\
\hline Complete response & 0 & 0 & 0 & & 0 & 0 & \\
\hline Partial response & 58 & 56 & 2 & & 10 & 48 & \\
\hline Stable disease & 52 & 50 & 2 & & 6 & 46 & \\
\hline Progressive disease & 42 & 40 & 2 & & 12 & 30 & \\
\hline N.E. & 1 & 1 & 0 & & 1 & 0 & \\
\hline Response rate & $37.9 \%$ & $38.1 \%$ & $33.3 \%$ & 0.950 & $34.5 \%$ & $38.7 \%$ & 0.202 \\
\hline Disease control rate & $71.9 \%$ & $72.1 \%$ & $66.7 \%$ & 0.915 & $55.2 \%$ & $75.8 \%$ & 0.020 \\
\hline
\end{tabular}

(A)

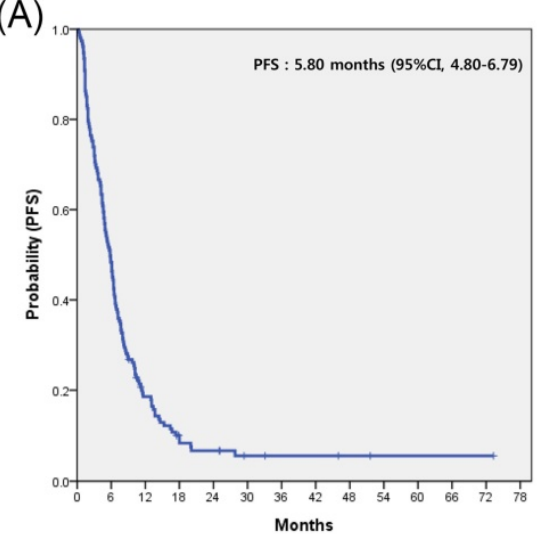

(B)

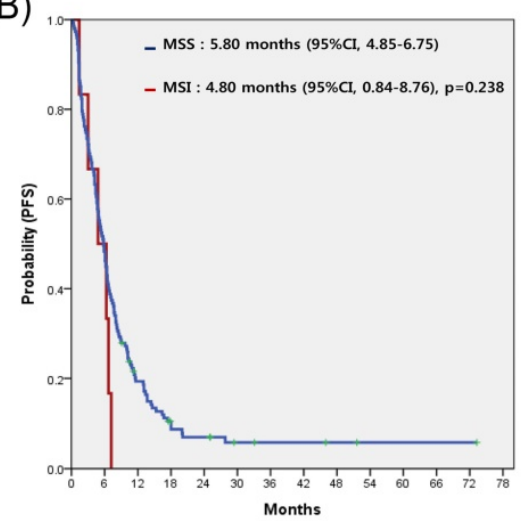

(C)

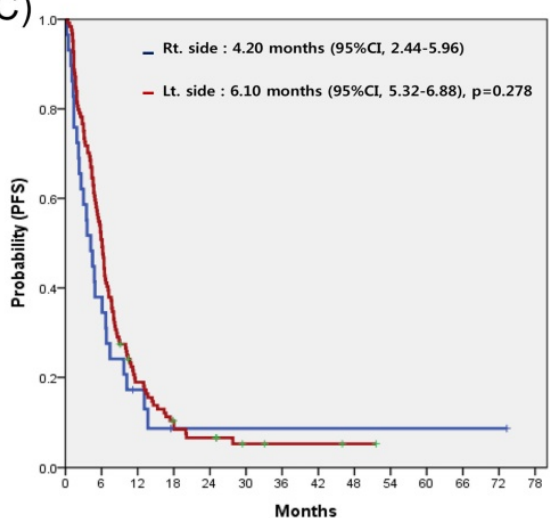

Figure 2. Progression-free survival (PFS) to cetuximab-containing chemotherapy in overall patients (A), according to the status of microsatellite instability (B), and the location of the primary tumor $(\mathrm{C})$.

(A)

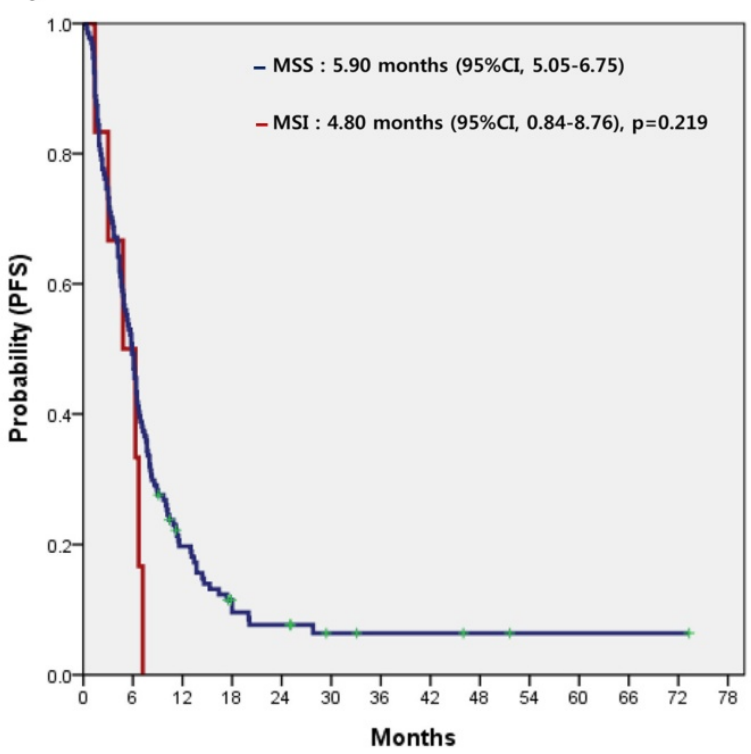

(B)

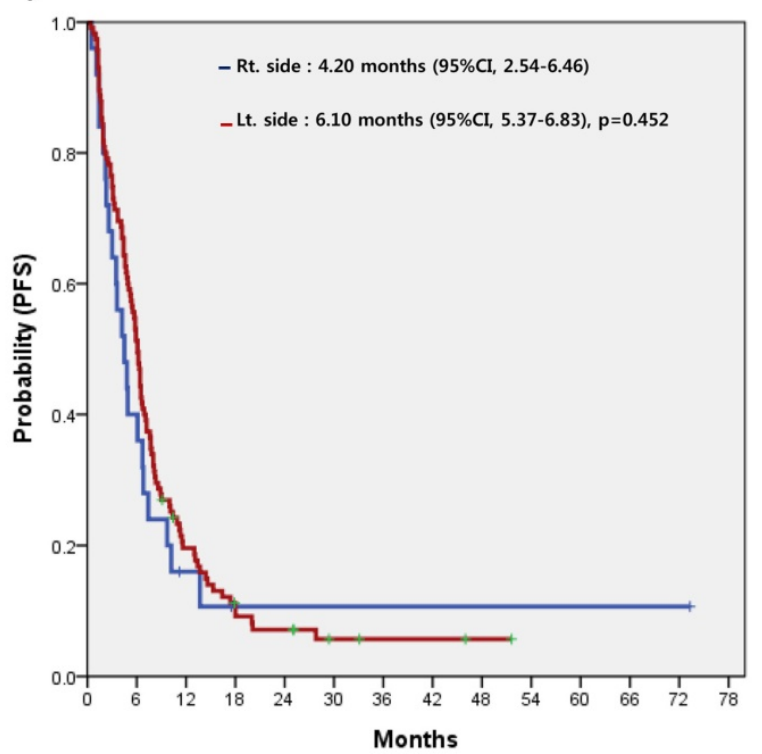

Figure 3. Progression-free survival (PFS) to cetuximab-containing chemotherapy in patients with wild-type KRAS, according to the status of microsatellite instability (A), and location of the primary tumor (B). 
Colorectal cancer (CRC) has been reconsidered as a heterogeneous disease. Because of the vastly different embryologic development of the left- and right-sided colon, in that the left colon originates from the hindgut and the right colon from the midgut, the luminal contents of the two sides are dissimilar [27, 28]. Accordingly, cancer of the left and right sides of the colon differs with respect to epidemiology, biology, microenvironment and clinical outcomes [27, 29-32]. However, it is unclear whether such differences in the two sides impact the effectiveness of targeted agents in colon cancer, because there is little evidence on the influence of primary tumor location in patients receiving cetuximab- or bevacizumab-containing therapy. Outcomes after cetuximab-containing therapy in left- and right-sided colon cancer have recently been reported. Analysis of the FIRE-3 trial comparing first line FOLFIRI with either cetuximab or bevacizumab showed that among patients with the KRAS wild type in the cetuximab arm, those with LC had significantly better PFS. This finding was validated in another analysis [33]. However, the present analysis found that tumor location was not predictive of a PFS benefit from cetuximab-containing therapy in patients with the KRAS wild type. This discrepancy may be due to differences in patient populations. In contrast with the other analyses, we included all patients receiving cetuximab-containing therapy irrespective of treatment-lines (Supplementary Table 1). Along with KRAS and BRAF, other epigenetic abnormalities, oncogenic alterations such as PIK3CA, NRAS, and other protein kinases may alter the effect of cetuximab [31, 34-38]. These comprehensive genetic alterations have not yet been in included in any studies. Such differences might underlie the inconsistent findings between the previous analysis and the present study. On analyzing PFS for patients with KRAS wild type receiving cetuximab-containing therapy as first line, PFS showed more favorable trend in LC rather than RC (LC: 10.9 months (7.13-14.67) vs. RC: 7.10 months (5.99-8.20), consistent to previous studies.

Table 4. Univariable progression-free survival analysis with proportional hazard regression in CRC patients with chemotherapy containing cetuximab.

\begin{tabular}{llll}
\hline Prognostic variable & Hazard ratio & $95 \% \mathrm{CI}$ & $\mathrm{p}$-value \\
\hline Age $(\leq 65$ yr vs. $>65$ yr) & 0.980 & $0.672-1.428$ & 0.915 \\
Gender (Male vs. Female) & 1.008 & $0.851-1.193$ & 0.930 \\
Primary site (Lt. side vs. Rt. side) & 1.264 & $0.825-1.936$ & 0.282 \\
No. of metastatic site (1 vs. $1<$ ) & 1.205 & $0.865-1.680$ & 0.270 \\
KRAS status (Wild vs. Mutant) & 1.331 & $0.751-2.360$ & 0.328 \\
BRAF status (Wild vs. Mutant) (n=72) & 1.029 & $0.442-2.395$ & 0.947 \\
Microsatellite instability (MSS vs. MSI) & 1.277 & $0.845-1.931$ & 0.245 \\
\hline
\end{tabular}

The present study included KRAS mutant patients. Although current guidelines only recommend starting anti-EGFR therapies such as cetuximab or panitumumab in patients with wild-type KRAS, past practice has permitted the use of cetuximab in patients with irinotecan-refractory CRC. In addition to patients with wild-type KRAS, we also analyzed all patients treated with cetuximab-containing therapy. This analysis revealed that tumor location was not predictive of a PFS benefit from cetuximab-containing therapy.

MSI-H colorectal cancers are known to bear many features that are generally associated with poor prognosis, including deep tumor invasion and poor histologic differentiation [12, 21, 22]. However, we couldn't evaluate the correlation between clinical features and MSI-H tumor because this study included only 6 MIS-H tumor patients.

MSI-H tumors are hypermutated and express abundant frameshift peptides that serve as neoantigens to elicit a brisk immune response characterized by abundant tumor-infiltrating lymphocytes [39]. In studies of adjuvant chemotherapy in stage II or III colon cancer, patients with MSI-H received no benefit from a regimen containing FU, unlike patients whose tumors demonstrate CIN [40-42]. However, the clinical impact of MSI status on treatment response to cetuximab-containing regimens in metastatic CRC has not been confirmed. This analysis showed that the efficacy of cetuximab-containing therapy was not statistically different between MSI-H and MSS tumors. Of course, this finding must be interpreted with caution. Due to inherent bias from a retrospective design with many confounding factors and the rather small sample size found in our study, findings in this study must be interpreted with caution. Further prospective validation of any prognostic or predictive capacity of MSI status should be performed.

Right and left-sided colon cancer is now recognized to have distinct clinical and genomic features. Similarly, MSI-H and MSS tumors are also regarded as two different heterogeneous entities. These heterogeneities must be reflected to stratify patients in order to realize a personalized medicine approach in CRC. In particular, cetuximab is considered an important treatment option in metastatic CRC. Following the discovery that mutations in KRAS are associated with resistance to anti-EGFR treatment, determination of KRAS status is now recommended in $\mathrm{mCRC}$ patients before starting anti-EGFR therapies. Despite the application of these selective strategies, less than half of the chosen wild-type KRAS patient population benefits from 


\section{anti-EGFR treatment.}

In the era of personalized medicine, being able to establish preferred treatment strategies based on RC versus LC and/or MSI-H versus MSS tumors may improve treatment outcomes and allow for more effective targeted therapy among CRC patients.

\section{Supplementary Material}

Supplementary table 1.

http://www.jcancer.org/v08p2809s1.pdf

\section{Acknowledgements}

This work was supported by funding from the Korean Health Technology R\&D Project, Ministry of Health \& Welfare, Republic of Korea (HI14C2750, HI14C3418). Support was also provided by a grant from Samsung Medical Center (SMX1161251).

\section{Competing Interests}

The authors have declared that no competing interest exists.

\section{References}

1. Jemal A, Bray F, Center MM, et al. Global cancer statistics. CA Cancer J Clin. 2011; 61: 69-90.

2. Cunningham D, Humblet $Y$, Siena $S$, et al. Cetuximab monotherapy and cetuximab plus irinotecan in irinotecan-refractory metastatic colorectal cancer. N Engl J Med. 2004; 351: 337-345.

3. de Gramont A, Figer A, Seymour M, et al. Leucovorin and fluorouracil with or without oxaliplatin as first-line treatment in advanced colorectal cancer. J Clin Oncol. 2000; 18: 2938-2947.

4. Hurwitz H, Fehrenbacher L, Novotny W, et al. Bevacizumab plus irinotecan, fluorouracil, and leucovorin for metastatic colorectal cancer. N Engl J Med. 2004; 350: 2335-2342.

5. Saltz LB, Cox JV, Blanke C, et al. Irinotecan plus fluorouracil and leucovorin for metastatic colorectal cancer. Irinotecan Study Group. N Engl J Med. 2000; 343: 905-914.

6. Van Cutsem E, Kohne $\mathrm{CH}$, Hitre E, et al. Cetuximab and chemotherapy as initial treatment for metastatic colorectal cancer. N Engl J Med. 2009; 360: 1408-1417.

7. Van Cutsem E, Peeters M, Siena S, et al. Open-label phase III trial of panitumumab plus best supportive care compared with best supportive care alone in patients with chemotherapy-refractory metastatic colorectal cancer. J Clin Oncol. 2007; 25: 1658-1664.

8. Heinemann V, von Weikersthal LF, Decker T, et al. FOLFIRI plus cetuximab versus FOLFIRI plus bevacizumab as first-line treatment for patients with metastatic colorectal cancer (FIRE-3): a randomised, open-label, phase 3 trial. Lancet Oncol. 2014; 15: 1065-1075.

9. Gervaz P, Bucher P, Morel P. Two colons-two cancers: paradigm shift and clinical implications. J Surg Oncol. 2004; 88: 261-266.

10. Gervaz P, Cerottini JP, Bouzourene H, et al. Comparison of microsatellite instability and chromosomal instability in predicting survival of patients with T3N0 colorectal cancer. Surgery. 2002; 131: 190-197.

11. Haydon AM, Jass JR. Emerging pathways in colorectal-cancer development. Lancet Oncol. 2002; 3: 83-88.

12. Jass JR, Whitehall VL, Young J, et al. Emerging concepts in colorectal neoplasia. Gastroenterology. 2002; 123: 862-876.

13. Aaltonen LA, Peltomaki P, Leach FS, et al. Clues to the pathogenesis of familial colorectal cancer. Science. 1993; 260: 812-816.

14. Ionov Y, Peinado MA, Malkhosyan S, et al. Ubiquitous somatic mutations in simple repeated sequences reveal a new mechanism for colonic carcinogenesis. Nature. 1993; 363: 558-561.

15. Soreide K, Janssen EA, Soiland $\mathrm{H}$, et al. Microsatellite instability in colorectal cancer. Br J Surg. 2006; 93: 395-406.

16. Miyakura Y, Sugano K, Akasu T, et al. Extensive but hemiallelic methylation of the hMLH1 promoter region in early-onset sporadic colon cancers with microsatellite instability. Clin Gastroenterol Hepatol. 2004; 2: 147-156.

17. Herman JG, Baylin SB. Gene silencing in cancer in association with promoter hypermethylation. N Engl J Med. 2003; 349: 2042-2054.

18. Herman JG, Umar A, Polyak K, et al. Incidence and functional consequences of hMLH1 promoter hypermethylation in colorectal carcinoma. Proc Natl Acad Sci U S A. 1998; 95: 6870-6875.
19. Gryfe R, Kim H, Hsieh ET, et al. Tumor microsatellite instability and clinical outcome in young patients with colorectal cancer. N Engl J Med. 2000; 342: 69-77.

20. Popat S, Hubner R, Houlston RS. Systematic review of microsatellite instability and colorectal cancer prognosis. J Clin Oncol. 2005; 23: 609-618.

21. Jass JR, Do KA, Simms LA, et al. Morphology of sporadic colorectal cancer with DNA replication errors. Gut. 1998; 42: 673-679.

22. Thibodeau SN, Bren G, Schaid D. Microsatellite instability in cancer of the proximal colon. Science. 1993; 260: 816-819.

23. Roepman P, Schlicker A, Tabernero J, et al. Colorectal cancer intrinsic subtypes predict chemotherapy benefit, deficient mismatch repair and epithelial-to-mesenchymal transition. Int J Cancer. 2014; 134: 552-562.

24. Sadanandam A, Lyssiotis CA, Homicsko $\mathrm{K}$, et al. A colorectal cancer classification system that associates cellular phenotype and responses to therapy. Nat Med. 2013; 19: 619-625.

25. Budinska E, Popovici V, Tejpar S, et al. Gene expression patterns unveil a new level of molecular heterogeneity in colorectal cancer. J Pathol. 2013; 231: 63-76.

26. De Sousa EMF, Wang X, Jansen M, et al. Poor-prognosis colon cancer is defined by a molecularly distinct subtype and develops from serrated precursor lesions. Nat Med. 2013; 19: 614-618.

27. Meguid RA, Slidell MB, Wolfgang CL, et al. Is there a difference in survival between right- versus left-sided colon cancers? Ann Surg Oncol. 2008; 15: 2388-2394.

28. Benedix F, Kube R, Meyer F, et al. Comparison of 17,641 patients with rightand left-sided colon cancer: differences in epidemiology, perioperative course, histology, and survival. Dis Colon Rectum. 2010; 53: 57-64.

29. Bufill JA. Colorectal cancer: evidence for distinct genetic categories based on proximal or distal tumor location. Ann Intern Med. 1990; 113: 779-788.

30. Fric P, Sovova V, Sloncova E, et al. Different expression of some molecular markers in sporadic cancer of the left and right colon. Eur J Cancer Prev. 2000; 9: 265-268.

31. Kalady MF, Dejulius KL, Sanchez JA, et al. BRAF mutations in colorectal cancer are associated with distinct clinical characteristics and worse prognosis. Dis Colon Rectum. 2012; 55: 128-133.

32. Wray CM, Ziogas A, Hinojosa MW, et al. Tumor subsite location within the colon is prognostic for survival after colon cancer diagnosis. Dis Colon Rectum. 2009; 52: 1359-1366.

33. Brule SY, Jonker DJ, Karapetis CS, et al. Location of colon cancer (right-sided versus left-sided) as a prognostic factor and a predictor of benefit from cetuximab in NCIC CO.17. Eur J Cancer. 2015; 51: 1405-1414.

34. De Roock W, Claes B, Bernasconi D, et al. Effects of KRAS, BRAF, NRAS, and PIK3CA mutations on the efficacy of cetuximab plus chemotherapy in chemotherapy-refractory metastatic colorectal cancer: a retrospective consortium analysis. Lancet Oncol. 2010; 11: 753-762.

35. Minoo P, Zlobec I, Peterson M, et al. Characterization of rectal, proximal and distal colon cancers based on clinicopathological, molecular and protein profiles. Int J Oncol. 2010; 37: 707-718.

36. Douillard JY, Oliner KS, Siena S, et al. Panitumumab-FOLFOX4 treatment and RAS mutations in colorectal cancer. N Engl J Med. 2013; 369: 1023-1034.

37. Mao C, Yang ZY, Hu XF, et al. PIK3CA exon 20 mutations as a potential biomarker for resistance to anti-EGFR monoclonal antibodies in KRAS wild-type metastatic colorectal cancer: a systematic review and meta-analysis. Ann Oncol. 2012; 23: 1518-1525.

38. Kim ST, Ahn TJ, Lee E, et al. Exploratory biomarker analysis for treatment response in KRAS wild type metastatic colorectal cancer patients who received cetuximab plus irinotecan. BMC Cancer. 2015; 15: 747.

39. Schwitalle $\mathrm{Y}, \mathrm{K}$ loor $\mathrm{M}$, Eiermann $\mathrm{S}$, et al. Immune response against frameshift-induced neopeptides in HNPCC patients and healthy HNPCC mutation carriers. Gastroenterology. 2008; 134: 988-997.

40. Sargent DJ, Marsoni S, Monges G, et al. Defective mismatch repair as a predictive marker for lack of efficacy of fluorouracil-based adjuvant therapy in colon cancer. J Clin Oncol. 2010; 28: 3219-3226.

41. Sinicrope FA, Foster NR, Thibodeau SN, et al. DNA mismatch repair status and colon cancer recurrence and survival in clinical trials of 5-fluorouracil-based adjuvant therapy. J Natl Cancer Inst. 2011; 103: 863-875.

42. Sinicrope FA, Foster NR, Yoon HH, et al. Association of obesity with DNA mismatch repair status and clinical outcome in patients with stage II or III colon carcinoma participating in NCCTG and NSABP adjuvant chemotherapy trials. J Clin Oncol. 2012; 30: 406-412. 